\title{
Effects of Traditional Srichiangmai dance on Balance and Mobility in the Elderly
}

\author{
M. Buransri', Y. Phanpheng ${ }^{2}$ \\ ${ }^{1}$ Faculty of Education, Loei Rajabhat University, Loei, Thailand \\ ${ }^{2}$ Faculty of Science and Technology, Loei Rajabhat University, Loei, Thailand
}

\author{
CORRESPONDING AUTHOR: \\ Yanyong Phanpheng \\ Faculty of Science and Technology \\ Loei Rajabhat University \\ Loei, Thailand 42000 \\ E-mail: yanyong.pha@|ru.ac.th
}

DOI:

10.32098/mltj.02.2021.02

LEVEL OF EVIDENCE: 1B

\begin{abstract}
SUMMARY
Background. Previous research indicates that slow movement dance exercise can stimulate function of core muscles effectively may improve physical fitness and balance among older adults.

Methods. This study investigated the effects of Traditional Srichiangmai dance on balance and mobility in the elderly. Ninety participants aged 60-75 years, the data was normally distributed, were recruited and randomly assigned into two groups: A Traditional Srichiangmai dance group (TSD) $(n=45)$ and a walking group (WG) $(n=45)$. TSD program comprised 15 postures, 4 rounds of dance techniques sessions of 30 minutes, 3 time per week over the course of 12 weeks, while the WG kept their walking exercise. Before and after the training, the tests of health-related physical fitness (HRF) variables composed of body composition, general physiology, balance and mobility performance, were assessed using standardized tests including the Functional reach test (FRT), Timed Up and Go Test, (TUG), 6-minute walk test Chair stand test and Arm curl test for 30 seconds.

Results. The results revealed that in both groups, Physiological variables including heart rate rest, systolic blood pressure decreased while $\mathrm{VO}_{2 \max }$ increased, higher than the pre-test values $(\mathrm{p} \leq 0.05)$. FRT and TUG was better for those in TSD compared to the WG. There was significant in the FRT $(\mathrm{p} \leq 0.001)$ for those in the dance group. TSD had faster movement, took shorter time and performed a better score in the TUG after training $(\mathrm{p} \leq 0.001)$.

Conclusions. After 12 weeks, Traditional Srichiangmai dance significantly improved balance and mobility among older community-dwelling as potentially prevent age-related mobility and balance decline as well as its related fall risk.
\end{abstract}

\section{KEY WORDS}

Traditional Srichiangmai dance; balance; mobility; elderly; physical fitness.

\section{BACKGROUND}

Presently, the medical development supports the people to live longer. The elderly's physical change affects physical functions including muscular strength, cardiorespiratory endurance and flexibility as well as other congenital diseases $(1,2)$. The rate of physical fitness decreased when ages increased; the elderly's balance also reduced (3). The physical performance reduces in functioning due to the reduction of mobility and balance. This causes the elderly's related fall risk. $(4,5)$. It is found that among those who were 65 or older had $28-35 \%$ of fall risk whereas those who were 70 or older had $32-42 \%$ of fall risk, especially for the female elderly. Moreover, the statistics related to injury proposed by the Department of Disease Control, the ministry of public health in 2019 showed that the cause of death from fall was found the second whereas the first cause of death was road accident $(6,7)$. The activity to promote exercise to enhance muscles strength will help improve balance ability and reduce risk of for approximately 19 percent (8). 
To enhance physical fitness with readiness and regular exercise can help reduce the risk of disease for the elderly and result in better physical fitness improvement heart function system work together efficiently (9). The researcher, addition, found that endurance training in elderly can increase heart muscles and strength, blood circulation in blood cells and blood flowing to heart better. When the amount of blood flowing to heart increased, physical organs will receive sufficient blood for functioning $(10,11)$. Moreover, regular exercise and continuation in the elderly will decrease heart rate and resistance of blood vessels which will result in the decrease of blood pressure $(12,13)$. Appropriate exercise for the elderly should be in form of aerobic with low impact within 20-30 minutes, 3-5 times a week in order to make the exercise most effective (14 - 16).

Different forms of aerobic dance exercise are very popular. It is the form of exercise that compose art, calisthenics, basic movement and dance step to be composed and applied to be a form of moment in music rhythm (17). Fon Ram or folk dance is mixed between movement in rhythm and the control of actions or postures. The pattern of dance needs the movement of all parts of the body to move slowly, then it helps improve all 3 systems of physical movement including central nerve system, sensory nerve system and muscular systems. These are good for core muscles which related to balance ability. The previous studies showed that slow movement exercise can stimulate function of core muscles effectively such as Waikru muaythai exercise (18), Tai Chi, Qigong (19, 20). Dance is a movement skill which is easy to control functional of balance. It is applied for exercise with beautiful postures with rhythm of slow movement to reduce impact. It is suitable for elderly. According to the field study in Pranprao sub-district, Srichaingmai district, Nongkai province, Thailand it was found that the community had their own dance postures as their identical uniqueness and the pattern of slow movement in rhythm which can be performed by all ages and genders, especially for the elderly. Consequently, this form of exercise is appropriate for being applied for the elderly in the community.

As mentioned earlier, the researcher had an idea to promote the beautiful culture along with physical and mental health and became interested in developing the model of aerobic exercise with low impact and folk-dance postures in rhythm of movement in folk music. This activity can link with the lifestyle of the local people and it is the way to conserve folk dance of the community to be promoted internationally. Consequently, the researcher was interested in studying the result of using folk dance for exercising which is called the activity in this research Traditional Srichiangmai dance to enhance physical fitness including physiological, balance ability and mobility which are all important for daily-life activates and improving sustainable quality of life.

\section{MATERIALS AND METHODS}

This research is a Quai-experimental research design with two group pretest-posttest design, certified and proved by The Research Ethics Review Committee for Research Involving Human Research Participants, on $13^{\text {th }}$ March 2017, project no. HE 0299/2560. The participants understood the details of practice during the experiment and signed consent letter to participate in the research.

\section{Participants}

The number of 90 participants were the older people residing in the elderly school Nongkai Province, males and females aged 60-75 years old, doing normal routines without having regular exercise. The simple random sampling technique was applying the balance and mobility abilities to rank and draw a random draw into two groups; the first group consisted of 45 participants performing Traditional Srichiangmai dance and the second group consisted of 45 participants performing walking exercise. The participants were signed the consent document to participate in the research. All participants needed to pass the questionnaires for examining their general health history and physical activity readiness questionnaire (PAR-Q) specifying that they did not have any diseases involving with muscles and nerve systems. In addition, they were all able to walk. On the other hand, the participants who could not participate throughout the research project will be removed from the experiment.

\section{Experimental training}

In this study, the researchers designed both patterns of exercise as parts of health promotion activity for the elderly in the school for the older people. Both groups exercised for 30 minutes, 3 times a week, totally 12 weeks. For the details and instruction of exercise, the experimental group performed Traditional Srichiangmai dance exercise including warm-up and stretching for 5 minutes, then began practicing 15 Traditional Srichiangmai dancing postures techniques, each poste 32 continuous tempo and repeated 4 rounds for 20 minutes and practiced static stretching for 5 minutes. For the control group, the participants performed walking exercise beginning with warm-up, static stretching for 5 minutes and then walking with normal speed for 20 minutes and then doing static stretching for 5 minutes. Both 
groups maintained an intensity of exercise $60-75 \%$ of maximum heart rate. There were sport scientists observing and suggesting the participants for correct positions and posturers as well as breathing methods during the time of training.

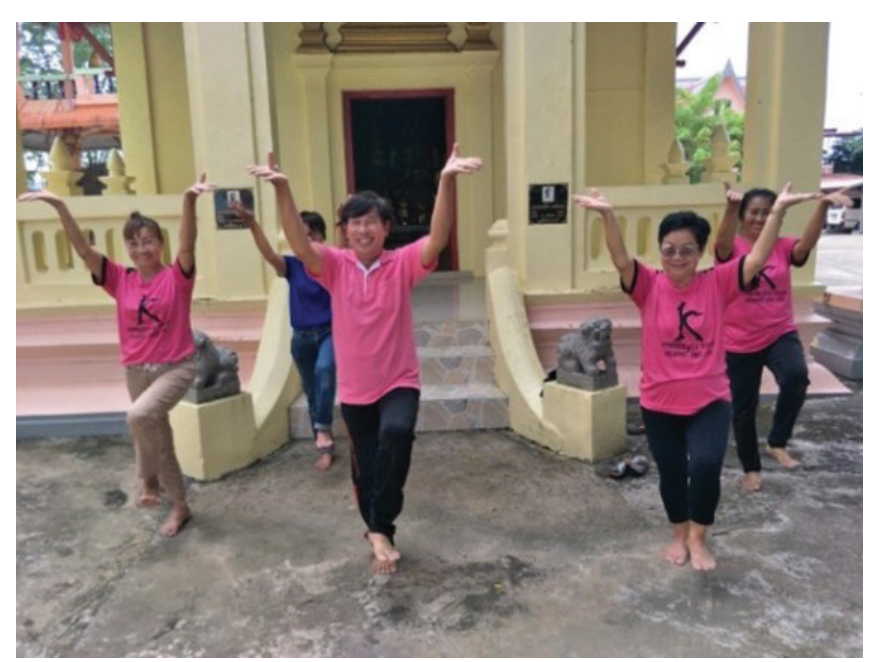

Figure 1. Tha Buachufack.

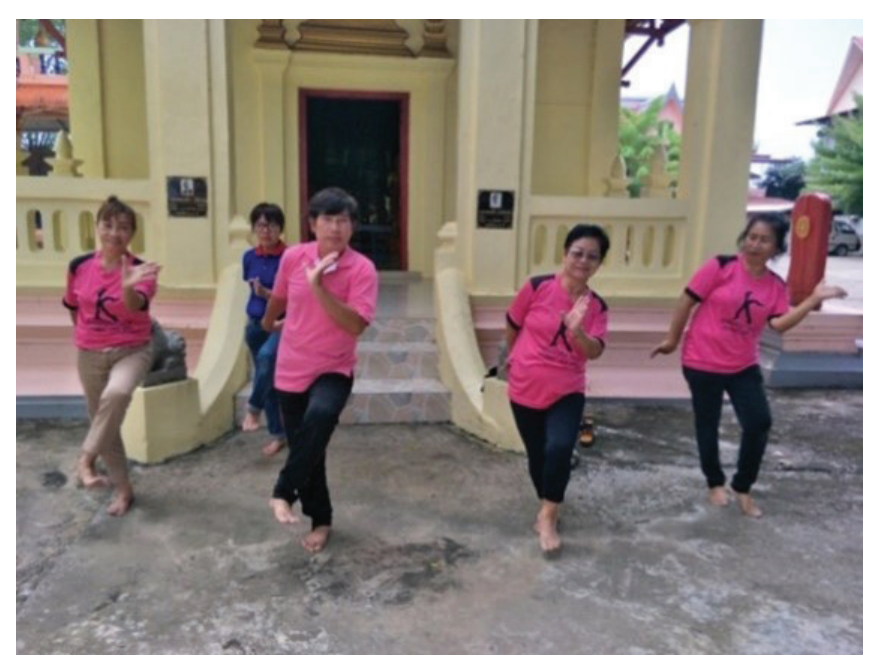

Figure 2. Tha Manee.

\section{Measurement of outcomes}

\section{Physiological}

The data collection was conducted to test the variables regarding health at Sport Science and Exercise Laboratory in the faculty of Science and Technology, Loei Rajabhat University, Thailand from 08.30-12.00 am. The samples performed rest sitting for 5 minutes and were measured with Omron HEM-7320 to examine their heart rate, systol- ic blood pressure and diastolic blood pressure. Then, the samples were examined for their body composition using Bioelectrical Impedance Analysis (BIA), in body 370 model, including body weight (kilogram) and IBM $\left(\mathrm{kg} / \mathrm{m}^{2}\right)$.

\section{Balance and mobility assessment}

The researcher evaluated the reliability within the measure (research assistant) to measure all two balance abilities before the actual assessment. The measurement reliability was excellent (ICC $=0.964,0.986$, respectively). Then, the balance was tested:

- method 1: in a standing position, arms were stretched at shoulder width, reaching forward (functional reach test, FRT) for as far as possible;

- method 2: the fluency and balance were tested while moving (agility and dynamic balance) by sitting, standing, walking (Timed Up and Go Test, TUG). Upon hearing the "start" signal, samples stood up from the chair, walked at as fast a distance as 8 feet, turned back, walked back and sat in the same chair.

The researchers measured the timer (seconds) from getting up from the chair and until returning to the chair. In addition, the researcher tested walking ability with 6-minute walk test, 60 meters for each of walking round, for 6 minutes. Chair stand test 30 second was conducted to test the strength and endurance of lower body test and the final test upper body muscles. The samples sat with arm curled doing arm curl test for 30 seconds then counted the number of times in a fully practiced posture.

Intensity control and Energy Expenditure; In both groups, intensity was controlled, $60-75 \%$ of maximum heart rate throughout the duration of each exercise. Intensity and energy expenditure while training with Traditional Srichiangmai dance and walking exercise group were observed. All participants put on heart rate monitor (Polar Team Pro) in which the signal was connected to the receiver (Apple Ipad). The results were displayed on the screen while training including Heart Rate (bpm), percent of Average Heart Rate $\left(\% A V G_{H R}\right)$, Percent of Maximum Heart Rate $\left(\% A V G_{\mathrm{HRmax}}\right)$ and Energy Expenditure of Exercise in Kilocalories (Kcal).

\section{Statistical analysis}

Descriptive statistics were used to describe the characteristics of the sample. A normal distribution test of data using the Kolmogorov-Smirnov Statistics Test $(\mathrm{n}>50)$ found that the data was normally distributed. Changes within the group were analysed before and after the training with the Paired t-test and the differences between the groups were 
Table I. Traditional Srichiangmai dance program.

\begin{tabular}{|c|c|c|c|}
\hline Day & Unit of training & Traditional Srichiangmai dance & Duration of training \\
\hline & Warm up & Static stretching of major muscle groups & 5 minutes \\
\hline \multirow[t]{2}{*}{$\begin{array}{l}\text { Monday Wednesday } \\
\text { Friday }\end{array}$} & Work out & $\begin{array}{l}\text { Tha Maesri } \\
\text { Tha Plangai } \\
\text { Tha Trawen weha } \\
\text { Tha Glang amporn } \\
\text { Tha Sodsoi mala } \\
\text { Tha Yonkeaw } \\
\text { Tha Pisamai riangmon } \\
\text { Tha Char nangnon } \\
\text { Tha Orashon } \\
\text { Tha Buachufack } \\
\text { Tha Kinaree } \\
\text { Tha On-an } \\
\text { Tha Srida } \\
\text { Tha Manee } \\
\text { Tha Nuaynard }\end{array}$ & $\begin{array}{l}15 \text { Traditional Srichiangmai } \\
\text { dancing postures techniques, } \\
\text { each poste } 32 \text { continuous tempo } \\
\text { and repeated } 4 \text { rounds } \\
\text { for } 20 \text { minutes }\end{array}$ \\
\hline & $\begin{array}{l}\text { Cool down and } \\
\text { stretching }\end{array}$ & Static stretching & 5 minutes \\
\hline
\end{tabular}

analysed with Independent t-test statistics at the significant level $\alpha=0.05$ and with SPSS 17.0 (SPSS Inc. Released 2008. SPSS Statistics for Windows, Version 17.0 Chicago: SPSS Inc.).

\section{RESULTS}

There were 90 volunteers in this study divided into two groups. However, the two volunteers disappeared during the experiment, in which each group did not complete the program as specified. Initially, the basic data analysis results of the volunteers in both groups were of average age. Most of the female groups in both groups were overweight referred to the BMI. The general data of the volunteers before exercise showed that there was no significant difference.
The analysis with pair t-test revealed that heart rate, systolic blood pressure and $\mathrm{VO}_{2 \max }$ of both groups had statistically significant difference when comparing between before and after exercise $(\mathrm{p} \leq 0.05)$. However, when analyzing between groups using Independent t-test statistics, using the mean values of change in physiological, there were no significant differences between the experimental groups. In addition, both groups were able to stabilize from the mobility of the lower body with the 6-minute walk test, strength and endurance of lower body with the chair stand test 30 second and upper body with the arm curl test for 30 second which was statistically improved within the group when comparing between before and after exercise $(p \leq 0.05)$. When analyzing between groups using Independent t-test statistics, using the mean of the change in balance ability. It was found that the experimental group exer-

Table II. General information of samples.

\begin{tabular}{|c|c|c|c|}
\hline & \multicolumn{2}{|l|}{ General information of samples } & \multirow[t]{2}{*}{$\mathbf{p}$} \\
\hline & $\begin{array}{l}\text { Experimental group }(n=45) \\
\text { Traditional Srichiangmai dance }\end{array}$ & $\begin{array}{l}\text { Control group }(n=45) \\
\text { Walking exercise }\end{array}$ & \\
\hline Gender (male:female) & $8: 37$ & $12: 33$ & - \\
\hline Age (year) & $63.64 \pm 4.6$ & $64.20 \pm 4.5$ & .641 \\
\hline Height $(\mathrm{cm})$ & $153.60 \pm 4.2$ & $153.84 \pm 4.0$ & .882 \\
\hline Weight $(\mathrm{kg})$ & $60.79 \pm 10.0$ & $61.26 \pm 9.5$ & .526 \\
\hline Body mass index $(\mathrm{kg} / \mathrm{m} 2)$ & $24.79 \pm 3.1$ & $24.81 \pm 2.9$ & .721 \\
\hline Average Heart Rate (\% AVGHR) & $67.21 \pm 12.9$ & $66.06 \pm 12.2$ & .825 \\
\hline Maximum Heart Rate (\% AVGHRmax) & $74.42 \pm 16.8$ & $74.10 \pm 16.1$ & .925 \\
\hline Expenditure of Exercise (Kcal) & $281.71 \pm 21.4$ & $280.20 \pm 21.4$ & .831 \\
\hline
\end{tabular}


Table III. Comparison of the mean values and standard deviations of the variables in Physiological, Balance and mobility between before and after 12-week experiment and control group.

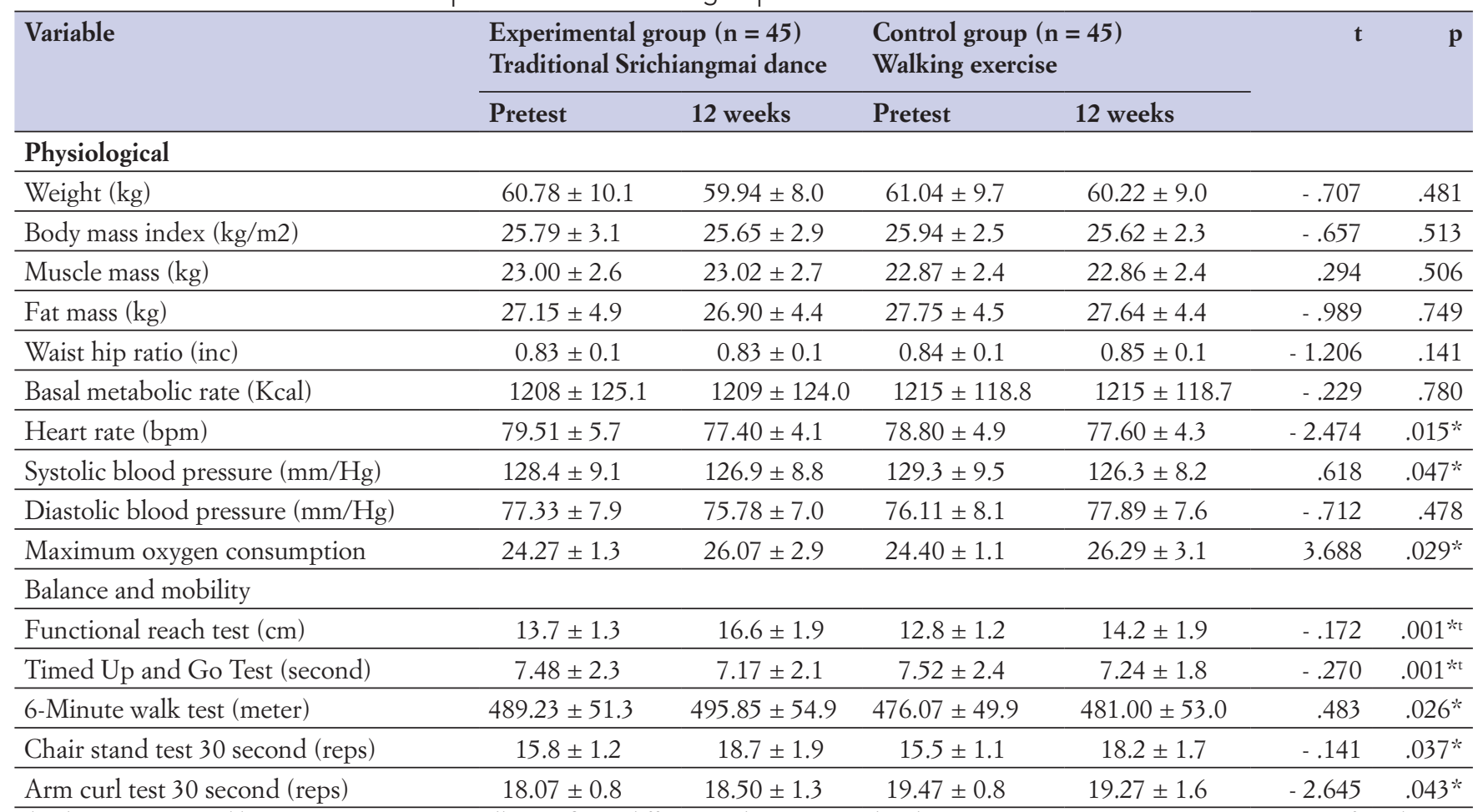

The data are presented by means \pm SD; * statistically significant difference when compared within group, mean scores at point comparisons from baseline: ${ }^{*} \mathrm{p} \leq 0.05$ and ${ }^{*} \mathrm{t} \leq 0.01$ when comparing the difference between experimental groups.

cising with respect to Traditional Srichiangmai dance had a statistically significant in their balance ability and mobility ( $\mathrm{p} \leq$ 0.001). Additionally, the functional reach test and timed up and go test (TUG) showed faster movement time, walking speed, and a better score before training.

\section{DISCUSSION}

This research was conducted by integrating the knowledge of the art of traditional dancing to create aerobic exercise forms of exercise that are novel, safe, fun, challenging, suitable for health promotion activities of the elderly. The exercise style emphasizes various movement skills, combining the Traditional Srichiangmai dance style which is the national cultural heritage of Thailand. And the researcher has studied the effect of exercise training on health-related physical fitness, balancing ability and mobility in the elderly by comparing with exercise styles by walking which is the introduction of both forms of exercise activities as part of promoting physical activities in the elderly school Nongkai, Thailand. The research findings can be discussed as follows.
The physiological data report of the two experimental groups after 12 weeks showed that the body composition, consisting of body weight, body mass index compared and fat mass to before the experiment has a tendency to decrease. There was no significant difference found after exercise. The change of variables related to body composition showed that both forms of exercise did activate of large muscles for continuous movement, and the circulation of all muscle groups. It can be said that this form of exercise is aerobic exercise, which is a process that uses the energy system of the body at a time level as well as the right weight to use energy from fat for combustion as energy, which is related to weight loss $(21,22)$. In addition, the side physiological variables were related to the cardiovascular system and the ability to work better. In this study, we found that after exercise, the heart rate and systolic blood pressure decreased when comparing to before exercise. According to the change, it can be explained that exercise that can maintain the level of heart rate and control the proportion of respiration provide a good effect on the heart, circulatory system and respiration. In this study, it was found that both subjects reduced in heart rate resting and systolic blood pressure. In addition, maximum oxygen consumption $\left(\mathrm{VO}_{2 \max }\right)$ increased 
when comparing to before exercise. The method of exercising with the Traditional Srichiangmai dance is using the rhythm of the music which has the relationship of the rhythm of body movement continuously. The participants were advised for how to breathe and manage fatigue while exercising. Those who practiced exercise can control the intensity of heart rate continuously. This is consistent with the recommendations of Emiliano et al. (23) who described that the benefits of breathing exercises while exercising can affect the efficiency of the breathing and the cardiac muscles that help with compression. This is related to the function of controlling the baroreceptor system. Exercise will stimulate the vagal tone, resulting in slower heart rate. Lower peripheral vascular resistance helps promote the function of the artery wall which is an important reason for the decrease in blood pressure values $(24,25)$.

Traditional Srichiangmai dance is a new alternative exercise that can be added physical fitness for the elderly. The basic parameters of the structure of the body that promotes good physical fitness are 2 components, which are the strength and endurance of the muscles which has a positive effect on the structure of the body. In this study, the researcher realized that the training posture with the basic movement skills in daily life of the elderly. From the 6-minute walk test chair stand test 30 second and arm curl test 30 second, it was found that both experimental groups had an increased average when compared with before the experiment with statistical significance at the level of 0.05 . In addition, the average post-exercise mean shows that Traditional Srichiangmai dance group have better balance ability and mobility with test the functional reach test (FRT) and movement time, walking speed with timed up and go test (TUG) than walking exercise groups when compared after 12 weeks of exercise. This is due to the use of Traditional Srichiangmai dance postures, using postures that use the power of the muscles of various parts of the body in rhythm, with the use of muscles in relation to the rhythm of the music that controls the movement. This helps control the muscles in various movements, postures and concentrate during practice. It is consistent to the study of Laophosri et al. (26) the study of exercise using Thai dance on the physical fitness and balance ability in the elderly. It was found that, after practicing Thai dance, there was a positive effect on balance ability. Similarly, Noopud et al. (27) described the benefits of Thai Dance to balance ability based on Berg balance score, strength of the muscles in lifting tests in the arms curl test for 30 seconds and Chair stand test for 30 seconds. The results are consistent to this result that Traditional Srichiangmai dance can help strengthen muscular strength which is a fundamental factor related to the ability to maintain a balanced body while moving effectively. In addition, Chen et al. (28) suggested that higher quadriceps strength was associated with better cognitive performance. This study focused on the study of variables related to balance in the elderly by the Functional reach test, which found that the samples had the ability to test the functional reach test in increasing range after exercise with statistical significance at the level of .001 . There is also the test of agility and dynamic balance with Timed Up and Go Test, which are basic skills in daily physical movements. From the test results, it was found that experimental group was able to improve their mobility within movement time, walking speed the body according to the conditions of the test with shorter duration. Although this study does not include mechanisms that increases the ability to balance performance directly, but it can discuss the strength from the designed posture training of Traditional Srichiangmai dance with rhythm. This pattern requires the principle of transferring weight during movement in rhythm with various postures together with weighting on the heel and toes which will stimulate the senses and maintain a more balanced weight on the feet $(2,29)$.

Aging is an inevitable biological process that is characterized by a general decline in the physiological and biochemical functions of the major systems. In the case of the neuromuscular system, reductions in strength and mobility cause a deterioration in motor performance, impaired mobility and disability (30). Traditional Srichiangmai dance was applied by the researcher using rhythm to control body movement together, due to the muscles are the effectors of the descending orders from the CNS, thus, the sensorial integration and its output to the muscles (31). Affect to contracting the abdominal muscles and lower back muscle to help increase the stability of the core muscle while moving rhythmically at all times while performing the strength of the core muscles that are responsible for controlling body movement $(32,33)$. This causes the motion recognition mechanism to work harmoniously (coordination movement) both the arms and legs, resulting in the control of the coordination of muscles (neuromuscular control) awareness of joints (proprioceptive sense) according to the rhythm repeatedly throughout the training period (34). That is to say, exercise in a muscle group that is primarily responsible for controlling the movement of the arms, legs, or core muscle groups, including abdominal muscles, back, pelvic floor and diaphragm, resulting better balance of the participants $(35,36)$. In this research, 15 Traditional Srichiangmai dancing postures techniques, each poste 32 continuous tempo and repeated 4 rounds of dance techniques were performed and repeatedly practiced with simple rhythm. Therefore, according to the above principles, it can be explained that the form of exercise by paying respect to Traditional Srichiangmai dance is to promote the mechanism of the relationship in the movement and develop the ability to maintain the balance of the body while in static (static balance) and the movement of the body (dynamic balance) as well as to practice learning and remem- 
bering. This is consistent with the recommendations of Rektorova et al. (37) an action observation, visuomotor integration and action imitation, that is activities that are all important for motor learning and executing skilled movements. It also has a reduce depression, medical expenses and use of medical facilities, and prevent falls (38). In the case of the elderly who are unable to comply, basic movements should be used. By starting from the easy posture to the difficult posture or begin by using a slow tempo and increasing the tempo faster when the participant can follow, local, regional music may be selected for familiarity and rhythm to have fun, enjoy, or use rhythmic instruments instead of music, with rhythms that can be easily remembered. Finally, in this research, the researchers working within the 24 field of clinical and sport science research, with a focus on the declaration of Helsinki (39) that 25 specified in the agreement in all respects.

\section{Suggestions for further research study}

It is suggested that the elderly should perform regular exercise with various forms of movement to stimulate blood circulation to muscular and nerve cells. This physical activity will enhance the increase of nerve cells creation and the relation with blood quantity flowing to Dentate gyrus of Hippocampus which is important for learning and remembering. In addition, it is essential for the development neural stem cells which is very interesting the study in the future.

\section{REFERENCES}

1. Maciaszek J. Effect of Tai Chi on Body Balance: Randomized Controlled Trial in Men with Osteopenia or Osteoporosis. Am J Chin Med 2007;35(1):1-9.

2. Yuan Z, Yan W. Tai Chi as an intervention to reduce falls and improve balance function in the elderly: a meta-analysis of randomized controlled trials. Chin Nurs Res 2016;30(3):28-33.

3. World Health Organization: WHO. Minimum Data Set Indicators for the minimum data set project on ageing: A critical review in sub-Saharan Africa 2001;42(2):122-6.

4. Christopher H, Kathryn LW, Shaun J, Matthew W. The effects of same-session combined exercise training on cardiorespiratory and functional fitness in older adults: a systematic review and meta-analysis. Aging Clin Exp Res 2019;31(3):1701-17.

5. Stec MJ, Thalacker MA, Mayhew DL. Randomized, fourarm, dose-response clinical trial to optimize resistance exercise training for older adults with agerelated muscle atrophy. Exp Gerontol 2017;26(2):98-109.

6. Matheus U, Chupel FD, Guilherme E, et al. Strength Training Decreases Inflammation and Increases Cognition and Physical Fitness in Older Women with Cognitive Impairment. Front Physiol 2017;12 (8):377

7. Adamo DE, Talley SA, Goldberg A. Age and task differences in functional fitness in older women: comparisons with senior

\section{CONCLUSIONS}

This Traditional Srichiangmai dance is an alternative aerobic exercise with slowly rhyming movement and low impact. This exercise is appropriate to be applied for exercise activity in the school for the old people in order to promote health related physical fitness. The results of the research revealed that Traditional Srichiangmai dance positively resulted in strength and endurance of lower body and improved core stability which are main factors for balance and effectiveness of responsive muscular structures as well as body movement control which is a basic skill for doing daily-life activities among the older people with confidence.

\section{ACKNOWLEDGMENTS}

Deeply thanks to all participants at the elderly school, Nongkai Province, Thailand everyone who cooperated well in this research. And this research was funded by the Thai Health Promotion Foundation, Research and Development Institute Loei Rajabhat University.

\section{CONFLICT OF INTERESTS}

The authors declare that they have no conflict of interests.

fitness test normative and criterion-referenced data. J Aging Phys Act 2015;23(3):47-54.

8. Health Information Systems Development Office. Promoting happy health for the elderly. J Health Doc 2019;11(3):115-22.

9. Hairi NN, Cumming RG, Naganathan V. Loss of muscle strength, mass (sarcopenia), and quality (specific force) and its relationship with functional limitation and physical disability: the concord health and ageing in men project. J Am Geriatr Soc 2010;58(1):2055-62.

10. Marsh SA, Coombes JS. Exercise and the endothelial cell. Int J Cardiol 2005;99(2):165-9.

11. Jo G, Rossow-Kimball B, Lee Y. Effects of 12-week combined exercise program on self-efficacy, physical activity level, and health related physical fitness of adults with intellectual disability. J Exerc Rehabil 2018;14(2):175-82.

12. Nybo L, Sundstrup E, Jakobsen MD, Mohr M, Hornstrup T, Simonsen L. High-Intensity Training Vs. Traditional exercise interventions for promoting health. Med Sci Sports Exerc 2010;74:225-36.

13. Shuang W, Hong TN, Su MX, et al. Effects of vibration therapy on muscle mass, muscle. J Phys Ther Sci 2020;28(2):1298-302.

14. 14. Donald HP, Gareth RJ, Charles LR. Ageing and physical activity: evidence to develop exercise recommendations for older adults. Can J Public Health 2007;98(2):70-84. 
15. Woo JS, Derleth C, Stratton JR, Levy WC. The influence of age, gender, and training on exercise efficiency. J Am Coll Cardiol 2006;47(4):1049-57.

16. Morris N, Gass G, Thompson M, Bennett G, Basic D, Morton $\mathrm{H}$. Rate and amplitude of adaptation to intermittent and continuous exercise in older men. Med Sci Sports Exerc 2002;34(2):471-7.

17. Panitjaroennam S. Aerobic Dance (Guide for leader) (5 $\left.5^{\text {th }} \mathrm{ed}\right)$. Bangkok print 2003.

18. Phanpheng Y, Larha W, Hiruntrakul A. Effects of wai khru muaythai training to balance ability in the elderly. Sport Sci Practical Aspects 2020;17(1):53-9.

19. Isaio I, Gil SM, Bidaurrazaga LI, Rodriguez LA. Effects of 3 months of detraining on functional fitness and quality of life in older adults who regularly exercise. Aging Clin Exp Res 2019;31(4):503-10.

20. Li JX, Hong Y, Chan KM. Tai chi: physiological characteristics and beneficial effects on health. Br J Sports Med 2001;35(3):148-56.

21. Otten L, Bosy WA, Ordemann J, et al. Abdominal fat distribution differently affects muscle strength of the upper and lower extremities in women. Eur J Clin 1 Nutr 2017;71(3):372-6.

22. Bouaziz W, Vogel T, Schmitt E. Health benefits of aerobic training programs in adults aged 70 and over: a systematic review. Arch Gerontol Geriatr 2017;69(3):110-7.

23. Emiliano AP, Vittorio P, Pasquale I, et al. Aerobic exercise performance correlates with post-ischemic flow-mediated dilation of the brachial artery in young healthy men. Eur J Appl Physiol 2005;94(2):113-7.

24. Marsh SA, Coombes JS. Exercise and the endothelial cell. Int J Cardiol 2005;99(2):165-9.

25. Carnelissen VA, Fagard RH. Effects of endurance training on blood pressure, blood pressure-regulating mechanism, and cardiovascular risk factors. Hypertension 2005;46(4):667-75.

26. Laophosri M, Kanpittaya J, Sawanyawisuth K, Auvichayapat P, Janyacharoen T. Effects of Thai dance on balance in Thai elderly. Chula Med J 2013;57(3):345-57.

27. Noopud P, Suputtitada A, Khongprasert S, Kanungsukkasem V. Effects of Thai traditional dance on balance perfor- mance in daily life among older women. Aging Clin Exp Res 2018;31(7):125-32

28. Chen WL, Peng TC, Sun YS, et al. Examining the Association Between Quadriceps Strength and Cognitive Performance in the Elderly. Medicine (Baltimore) 2015;94(32):e1335.

29. Nicole K, Michael AT. Core Muscle Strengthening's Improvement of Balance Performance in Community-Dwelling Older Adults: A Pilot Study. J Aging Phys Act 2014;22(1):65-73.

30. Buonocore D, Rucci S, Vandoni M, Negro M, Marzatico F. Oxidative system in aged skeletal muscle. Muscles Ligaments Tendons J 2011;1(3):85-90.

31. Andrade HB, Costa SM, Pirôpo US, Schettino L, Casotti CA, Pereira R. Lower limb strength, but not sensorial integration, explains the age-associated postural control impairment. Muscles Ligaments Tendons J 2017;7(4):598-602.

32. Kwon YK. Effects of core muscle stability training on the weight distribution and stability of the elderly. J Phys Ther Sci 2015;27(10):3163-5.

33. 33. Komagata S, Newton R. The Effectiveness of Tai Chi on Improving Balance in Older Adults: An Evidence-based Review. J Geriatr Phys Ther 2003;26(2):9-16.

34. 34. Kim YM, Park JH. The effect of whole-body vibration exercise on balance, muscle strength and falls efficacy in the elderly. J Korean Soc Phys Med 2017;12(4):61-71.

35. Sofianidis G, Hatzitaki V, Douka S, et al. Effect of a 10-week traditional dance program on static and dynamic balance control in elderly adults. J Aging Phys Act 2009;17(3):167-80.

36. Cho WS, Park CB, Lim JH. The effect of trunk strengthening exercise using oscillation on trunk muscle thickness and balance. J Korean Soc Phys Med 2017;12(1):91-101.

37. Rektorova I, Klobusiakova P, Balazova Z, et al. Brain structure changes in nondemented seniors after six-month dance-exercise intervention. Acta Neurol Scand 2020;141:90-7.

38. Jeon MY, Bark ES, Lee EG, Im JS, Jeong BS, Choe ES. Taehan Kanho Hakhoe Chi 2005;35(7):1268-76

39. Padulo J, Oliva F, Frizziero A, Maffulli N. Muscle, Ligaments and Tendons Journal. Basic principles and recommendations in clinical and field science research: 2018 update. Muscle Ligaments Tendons J 2018;8(3):305-7. 